\title{
Diagnostic accuracy of routine antenatal determination of fetal $R H D$ status across gestation: population based cohort study
}

\author{
() $(1) \Theta$ OPEN ACCESS
}

\author{
Lyn S Chitty professor ${ }^{1}$, Kirstin Finning clinical scientist ${ }^{2}$, Angela Wade senior lecturer ${ }^{3}$, Peter \\ Soothill professor ${ }^{4}$, Bill Martin consultant obstetrician ${ }^{5}$, Kerry Oxenford research midwife ${ }^{6}$, Geoff \\ Daniels consultant clinical scientist ${ }^{2}$, Edwin Massey associate medical director ${ }^{2}$
}

${ }^{1}$ UCL Institute of Child Health, Great Ormond Street Hospital for Children NHS Foundation Trust and University College London Hospitals NHS Foundation Trust, London WC1N 3BH, UK; ${ }^{2}$ International Blood Group Reference Laboratory, NHS Blood and Transplant, Bristol BS34 7QH, UK Kirstin Finning; ${ }^{3}$ Centre for Paediatric Epidemiology and Biostatistics, UCL Institute of Child Health, London WC1N 1EH, UK; ${ }^{4}$ Fetal Medicine Unit, St Michael's Hospital, Bristol BS2 8EG, UK; ${ }^{5}$ Fetal Medicine Unit, Birmingham Women's NHS Foundation Trust, Birmingham B15 2TG, UK; ${ }^{6} \mathrm{Fetal}$ Medicine Unit, University College London Hospitals NHS Foundation Trust, London NW1 2BU, UK

\begin{abstract}
Objectives To assess the accuracy of fetal RHD genotyping using cell-free fetal DNA in maternal plasma at different gestational ages.

Design A prospective multicentre cohort study.

Setting Seven maternity units in England.

Participants RhD negative pregnant women who booked for antenatal care before 24 weeks' gestation.

Interventions Women who gave consent for fetal RHD genotyping had blood taken at the time of booking for antenatal care and, when possible, at other routine visits such as for Down's syndrome screening between 11 and 21 weeks' gestation, at the anomaly scan at 18-21 weeks, and in the third trimester when blood was taken for the routine antibody check. The results of cord blood analysis, done routinely in $\mathrm{RhD}$ negative pregnancies, were also obtained to confirm the fetal $R H D$ genotyping

Main outcome measures The accuracy of fetal RHD genotyping compared with RhD status predicted by cord blood serology.

Results Up to four analyses per woman were performed in 2288 women, generating 4913 assessable fetal results. Sensitivity for detection of fetal $R H D$ positivity was $96.85 \%$ (94.95\% to $98.05 \%$ ), $99.83 \%$ (99.06\% to $99.97 \%$ ), $99.67 \%$ (98.17\% to $99.94 \%$ ), $99.82 \%$ (98.96\% to $99.97 \%$ ), and $100 \%(99.59 \%$ to $100 \%)$ at $<11,11-13,14-17,18-23$, and $>23$ completed weeks' gestation, respectively. Before 11 weeks' gestation $16 / 865$ (1.85\%) babies tested were falsely predicted as $R H D$ negative.

Conclusions Mass throughput fetal $R H D$ genotyping is sufficiently accurate for the prediction of RhD type if it is performed from 11 weeks' gestation. Testing before this time could result in a small but significant number of babies being incorrectly classified as $R H D$ negative. These mothers would not receive anti-RhD immunoglobulin, and there would
\end{abstract}

be a risk of haemolytic disease of the newborn in subsequent pregnancies.

\section{Introduction}

Haemolytic disease of the fetus and newborn is caused by maternal IgG antibodies to red cell surface antigens crossing the placenta and facilitating the immune destruction of fetal red cells or erythroid progenitors. The most commonly implicated antigen is the $\mathrm{RhD}$ (RH1) antigen of the rhesus system. Postnatal prophylaxis with anti-RhD immunoglobulin substantially reduces the alloimmunisation of $\mathrm{RhD}$ negative women and the prevalence of haemolytic disease of the fetus and newborn. Subsequent introduction of routine antenatal prophylaxis with anti-RhD immunoglobulin in the third trimester for all $\mathrm{RhD}$ negative pregnant women is now standard in many countries. ${ }^{2}$ In a predominantly white population, however, about $38 \%$ of these women would be carrying an $\mathrm{RhD}$ negative fetus and thus receive anti-RhD immunoglobulin, a pooled human plasma product, unnecessarily. ${ }^{3}$ Prophylaxis after delivery is offered only to $\mathrm{RhD}$ negative women who have given birth to an $\mathrm{RhD}$ positive baby.

Since the identification of cell-free fetal DNA in the blood of pregnant women, ${ }^{4}$ several laboratories have provided a fetal $R H D$ genotyping service for $\mathrm{RhD}$ negative women with a measureable concentration of anti-RhD antibody. ${ }^{5}$ Cell-free fetal DNA in maternal plasma is tested for the presence or absence of the $R H D$ gene, and results are used to direct management of the pregnancy. ${ }^{5}$

Consequent to the success of fetal $R H D$ genotyping in high risk women, the National Institute for Health and Care Excellence 
has "endorsed studies into the feasibility of mass testing antenatally for fetal blood group by analysis of cell-free fetal DNA in maternal plasma." ${ }^{2}$ If fetal $R H D$ testing proved accurate enough, there would be no need to test cord red cells serologically for $\mathrm{RhD}$ status.

High risk alloimmunised women are tested with a labour intensive low throughput method, which is unsuitable for mass screening. ${ }^{6}$ Two studies have used high throughput methods, including robotic isolation of plasma DNA and real time quantitative polymerase chain reaction (PCR) technology, to routinely determine fetal $R H D$ status at 30 weeks in the Netherlands ${ }^{7}$ and at 28 weeks in the United Kingdom, ${ }^{8}$ with 99.4\% and $95.7 \%$ diagnostic accuracy when compared with cord blood serology. If the results of the UK trials had been applied as a guide to treatment, only $2 \%$ of the women would have received anti-RhD immunoglobulin unnecessarily, compared with $38 \%$ in the absence of genotyping. Similar accuracy was achieved in validation studies in Germany ${ }^{9}$ and France, ${ }^{10}$ but the latter involved re-testing all negative results, which would be uneconomical. ${ }^{10}$

In Denmark and the Netherlands routine fetal RHD genotyping has recently been introduced as a national service at 26 and 28 weeks, respectively. ${ }^{112}$ As routine anti-RhD prophylaxis is recommended from 28 weeks, and anti-RhD immunoglobulin is administered earlier in pregnancy after any potential sensitising event, implementation of fetal RHD testing earlier in pregnancy would maximise potential benefits and cost reduction. As concentrations of cell-free fetal DNA in maternal blood increase throughout the pregnancy, ${ }^{13}$ it cannot be assumed that tests will be as accurate early in pregnancy as they are at 26-28 weeks' gestation.

We estimated the diagnostic accuracy and costs of fetal $R H D$ genotyping in $\mathrm{RhD}$ negative pregnant women from the time of booking until 28 weeks' gestation to determine the optimal gestation for implementation into routine clinical practice. The economic impact of testing will be reported separately.

\section{Methods \\ Population}

Women booking for antenatal care in 2009-12 at seven maternity units in England were prospectively asked for verbal consent to donate an additional $4 \mathrm{~mL}$ of blood at the time of their booking appointment. These appointments were in hospital antenatal clinics or the community according to local clinical practice. Local midwives got verbal consent from women when possible. Blood samples from RhD positive women were discarded, and those from $\mathrm{RhD}$ negative women were sent to the International Blood Group Reference Laboratory for fetal RHD genotyping, to arrive within 48 hours of blood being drawn. When possible, we also obtained consent to collect additional blood samples opportunistically for fetal $R H D$ genotyping from $\mathrm{RhD}$ negative women with singleton pregnancies when they attended for Down's syndrome screening (at 11-20 weeks) and the routine anomaly scan (at 18-23 weeks) (fig $1 \Downarrow$ ). While it is recommended that all women book for antenatal care early in pregnancy, before the offer of the combined screening test for Down's syndrome, which is performed at 11-13 weeks and 6 days, not all do. For the purpose of our study we included only women who booked before 24 weeks' gestation, agreed to participate in the study, and donated at least one blood sample before 24 weeks' gestation. Those who booked before 11 weeks might have donated up to four blood samples: at booking; at the time of screening for Down's syndrome (either the combined test at 11-13 weeks and 6 days or second trimester screening at 14-20 weeks); at the routine anomaly scan, which, although recommended at 18-21 weeks and 6 days, can be performed as late as 24 weeks in some circumstances; and after 25 weeks at the routine third trimester antenatal visit, when blood is taken to check haemoglobin and antibody concentrations.

Written informed consent was obtained before or at the routine third trimester visit (around 28 weeks), when the women were given the result of molecular testing and an additional blood sample was requested to confirm fetal $R H D$ status. When third trimester testing confirmed that the fetus was $R H D$ negative, women were allowed to opt out of receiving anti-RhD immunoglobulin if they requested to do so as the reliability of testing at this gestation is proved. ${ }^{811}$ All women predicted to be carrying an $\mathrm{RhD}$ positive baby, or when the fetal genotyping result was inconclusive, were offered anti-RhD immunoglobulin according to local clinical practice. All women experiencing a sensitising event before 28 weeks' gestation were also offered anti-RhD immunoglobulin according to local clinical practice.

\section{Fetal genotyping from maternal blood}

On receipt of blood samples, plasma was prepared and analysed prospectively by using assay techniques and criteria for the determination of fetal $R H D$ status as described previously.

\section{Serological RHD testing on cord bloods}

$\mathrm{RhD}$ typing on cord blood samples was performed with serological methods routinely used by participating units. Results were ascertained by the research midwives and compared with the genotype predicted.

\section{Data analysis}

We included results obtained from $\mathrm{RhD}$ negative women before 25 weeks with a singleton pregnancy who gave written consent for fetal $R H D$ genotyping and in whom a cord blood $\mathrm{RhD}$ group was subsequently obtained. Genotyping results were interpreted as $R H D$ positive, $R H D$ negative, or inconclusive. Sensitivity for the detection of $R H D$ positivity was calculated on the basis that inconclusive results were treated as $R H D$ positive. We used generalised linear models with random intercept to quantify trends in sensitivity and specificity with gestational age while taking into account repeat measures from the same woman. All estimates are presented with $95 \%$ confidence intervals. We used $\mathrm{R}$ (version 2.14.0) library glmmML to fit the models with 5000 bootstrap samples for estimation of standard errors.

\section{Results}

Women were recruited from 2009 to 2012. Of the samples received at the International Blood Group Reference Laboratory, 172 were excluded from the analysis because of extreme haemolysis (average transit time 8.5 days), and a further 22 were not analysed because they did not contain sufficient blood. Samples from 30 women were excluded because of multiple pregnancy, 185 because no cord blood result was subsequently obtained, and 372 because of lack of written consent. We included results of fetal $R H D$ genotyping from 2288 women in the final analysis. We had 4913 fetal genotype results with up to four analyses per woman; 436 had only one measurement, 1132 had two, 667 had three, and 53 had four. Most women were white (78\%), 5.8\% were Asian, $3.9 \%$ were black or mixed race, and $12.3 \%$ were unknown. Gestational age at measurement varied from five to 35 weeks (mean 18.67, median 19). 
Genotyping results were inconclusive for 393/4913 (8\%, 95\% confidence interval $7.27 \%$ to $8.79 \%$ ) of the samples (table $1 \Downarrow$ ). Analysis of cord blood showed that 2023 samples were from $\mathrm{RhD}$ negative babies, of which 18 had been falsely predicted antenatally to be genotypically $R H D$ positive and 85 had been inconclusive; 2890 were $\mathrm{RhD}$ positive, of which 308 had been inconclusive on fetal $R H D$ genotyping and 19 were falsely predicted genotypically to be $R H D$ negative (table 1 ).

The odds of correctly identifying $R H D$ positive and $R H D$ negative fetuses increased significantly with gestational age, with low levels of false negative results after 11 weeks' gestation. Table 1 shows the summary statistics for $<11,11-13$, 14-17, 18-23, and $>23$ weeks $\Downarrow$. Figure $2 \Downarrow$ shows how sensitivity for correctly identifying $R H D$ positive fetuses increased on a week by week basis and how before 11 weeks, confidence intervals were wider and sensitivities not good enough to support routine introduction of the test.

\section{Discussion}

Previous studies have shown that mass throughput $R H D$ genotyping of cell-free fetal DNA is accurate if performed in the third trimester of pregnancy. ${ }^{78}$ In this population based cohort study, we evaluated accuracy across a wider range of gestations and have shown that it is highly accurate from 11 weeks' gestation; before this the percentage of babies falsely predicted as $\mathrm{RhD}$ negative increases. For routine application of fetal $R H D$ genotyping, false negative results - whereby the fetus is predicted to be $\mathrm{RhD}$ negative when it is really $\mathrm{RhD}$ positive-pose the greatest concern as mothers will not receive prophylactic anti-RhD immunoglobulin and might alloimmunise, which would put babies in subsequent pregnancies at risk of considerable morbidity or mortality. As the sensitivity improves with gestation, this risk can therefore be mitigated by optimal timing of testing, by repeating cell-free fetal DNA testing in women predicted to be carrying an $\mathrm{RhD}$ negative baby, or by testing cord blood of babies predicted to be $\mathrm{RhD}$ negative. The latter two interventions would, however, impact on the cost effectiveness and applicability of a large scale programme. In our study, the incidence of false $R H D$ negative results before 11 weeks' gestation was $16 / 865$ (1.85\%, $95 \%$ confidence interval $1.14 \%$ to $2.98 \%$ ). This might be because of low concentrations of cell-free fetal DNA in early pregnancy. ${ }^{13}$ After 11 weeks the incidence of false negative results was considerably lower (at 1/956 between $11-13$ weeks; $0.1 \%, 0.02 \%$ to $0.59 \%$ ) and did not decrease between 11 and 24 weeks, in line with recent evidence that concentrations of cell free fetal DNA increase only minimally between 10 and 20 weeks' gestation. ${ }^{14}$ The small number of false $R H D$ negative results after 11 weeks' gestation would only minimally increase the risk of alloimmunisation in the population, around 0.07/100 000 births (table $2 \Downarrow$ ). This figure could well be offset by the potential to improve overall uptake of the immunoprophylaxis programme for $\mathrm{RhD}$ negative women carrying an $R H D$ positive baby, as counselling and follow-up could be targeted at these women to try to improve anti-RhD immunoglobulin uptake when it is definitively needed.

In Denmark ${ }^{11}$ and the Netherlands ${ }^{12}$ routine fetal $R H D$ genotyping has been introduced at 25 and 28 weeks' gestation, respectively, and is used to direct routine third trimester antenatal prophylaxis with anti-RhD immunoglobulin. While such strategies can minimise the administration of unnecessary anti-RhD immunoglobulin in a routine immunoprophylaxis programme, they do not take account of anti-RhD immunoglobulin required for sensitising events earlier in pregnancy. Earlier knowledge of fetal $R H D$ genotype would further reduce unnecessary administration of anti-RhD immunoglobulin as well as potentially enhancing any financial savings as prophylactic anti-RhD immunoglobulin and costly estimations of fetomaternal haemorrhage by Kleihauer-Betke test or flow cytometry could be avoided in $\mathrm{RhD}$ negative mothers carrying an $R H D$ negative baby, savings realised not only for routine prophylaxis at 28 weeks but also for sensitising events occurring at earlier gestations. ${ }^{15} 16$

\section{Conclusions}

Mass throughput fetal RHD genotyping is sufficiently accurate to be used from 11 weeks' gestation. To maximise reduction in administration of anti-RhD immunoglobulin and to minimise costs to both women and health services, testing should be performed as soon as possible from 11 weeks' gestation to coincide with routine antenatal visits.

We acknowledge the assistance of Rekha Anand, NHS Blood and Transplant, Birmingham, UK; and the research midwives and medical and laboratory staff at Birmingham Women's NHS Foundation Trust Hospital; St Michael's Hospital Bristol, North Bristol Trust, Bristol; University College London Hospitals NHS Foundation Trust; Royal Victoria Hospital, Newcastle upon Tyne; and City Hospitals Sunderland NHS Foundation Trust.

Contributors: LSC, GD, KF, EM, AW, and PS designed the study; LSC, $E M$, and GD carried out the literature search; AW created the figures; $\mathrm{KF}$, PS, BM, and KO collected the data; AW analysed the data; LSC, GD, KF, EM, and AW interpreted the data; LSC, EM, GD, and AW wrote the paper, which was revised by all authors. LSC is guarantor.

Funding: This study was funded by the National Institute for Health Research, Research for Patient Benefit Programme (PB-PG-0107-12005), and Howard Ostin Trust (Project No 011). LSC is partially funded by the Great Ormond Street Hospital Children's Charity and the NIHR Biomedical Research Centre at Great Ormond Street Hospital. The funded research is independent and the views expressed in the paper are those of the authors and not necessarily those of the NHS, the NIHR, or the Department of Health.

Competing interests: All authors have completed the ICMJE uniform disclosure form at www.icmje.org/coi_disclosure.pdf and declare: no support from any organisation for the submitted work; no financial relationships with any organisations that might have an interest in the submitted work in the previous three years; no other relationships or activities that could appear to have influenced the submitted work.

Ethical approval: The study was approved by the joint UCL/UCLH committee on the ethics of human research (committee A) of the National Research Ethics Service (07/H0714/128).

Transparency: The lead author affirms that the manuscript is an honest, accurate, and transparent account of the study being reported and that no important aspects of the study have been omitted.

Data sharing: No additional data are available.

1 Kumar S, Regan F. Management of pregnancies with RhD alloimmunisation. BMJ 2005;330:1255-8.

2 National Institute for Health and Care Excellence. Technology appraisal guidance 41 Guidance on the use of routine antenatal anti-D prophylaxis for RhD-negative women. NICE, 2002.

3 Daniels G. Human blood groups. 3rd ed. Wiley-Blackwell Science, 2013.

4 Lo YM, Corbetta N, Chamberlain PF, Rai V, Sargent IL, Redman CW, et al. Presence of fetal DNA in maternal plasma and serum. Lancet 1997;350:485-7.

5 Daniels G, Finning K, Martin P, Massey E. Non-invasive prenatal diagnosis of fetal blood group phenotypes: current practice and future prospects. Prenat Diagn 2009;29:101-7.

$6 \quad$ Finning K, Martin P, Daniels G. A clinical service in the UK to predict fetal Rh (Rhesus) $\mathrm{D}$ blood group using free fetal DNA in maternal plasma. Ann N Y Acad Sci 2004;1022:119-23.

7 Van der Schoot CE, Soussan AS, Koelewijn J, Bonsel G, Paget-Christiaens LG, de Haas M. Non-invasive antenatal RHD typing. Transfus Clin Biol 2006;13:53-7. 


\section{What is already known on this topic}

Previous studies have predominantly assessed accuracy of fetal $R H D$ genotyping at relatively late gestations (24-30 weeks)

At this stage in pregnancy it is highly accurate and can be used to direct immunoprophylaxis to those RhD negative women carrying an $R H D$ positive baby and thereby reduce unnecessary exposure to anti-RhD immunoglobulin

\section{What this study adds}

Mass throughput fetal $R H D$ genotyping is highly accurate from 11 weeks' gestation, but before this the number of babies falsely predicted to be $\mathrm{RhD}$ negative is high

To maximise benefit of routine fetal $R H D$ genotyping and to avoid potential sensitisation by withholding anti-RhD immunoglobulin in women falsely predicted to be carrying an RhD negative baby, testing should be done only from 11 weeks' gestation

8 Finning K, Martin P, Summers J, Massey E, Poole G, Daniels G. Effect of high throughput RHD typing of fetal DNA in maternal plasma on use of anti-RhD immunoglobulin in RhD negative pregnant women: prospective feasibility study. BMJ 2008;336:816-8.

9 Müller SP, Bartels I, Stein W, Emons G, Gutensohn K, Kohler M, et al. The determination of the fetal $\mathrm{D}$ status from maternal plasma for decision making on $\mathrm{Rh}$ prophylaxis is feasible. Transfusion 2008:48:2292-301.

10 Rouillac-Le Sciellour C, Puillandre P, Gillot R, Baulard C, Metral S, Le Van Kim C, et al. Large-scale pre-diagnosis study of fetal RHD genotyping by PCR on plasma DNA from RhD-negative pregnant women. Mol Diagn 2004;8:23-31.

11 Clausen FB, Christiansen M, Steffensen R, Jorgensen S, Nielsen C, Jakobsen MA, et al. Report of the first nationally implemented clinical routine screening for fetal RHD in Dpregnant women to ascertain the requirement for antenatal $\mathrm{RhD}$ prophylaxis. Transfusion 2012;52:752-8.

12 De Haas M, van der Ploeg CPB, Scheffer PG, Verlinden DA, Hirschberg H, Abbink F, et al. A nationwide fetal RHD screening programme for targeted antenatal and postnatal anti-D. ISBT Sci Ser 2012;7:164-7.

13 Lun FM, Chiu RW, Chan KC, Leung TY, Lau TK, Lo YM. Microfluidics digital PCR reveals a higher than expected fraction of fetal DNA in maternal plasma. Clin Chem 2008;54:1664-72.
14 Wang E, Batey A, Struble C, Musci T, Song K, Oliphant A. Gestational age and maternal weight effect on fetal cell-free DNA in maternal plasma. Prenat Diagn 2013;33:662-6.

15 Qureshi H, Massey E, Kirwan D, Davies T, Robson S, White J, et al. BCSH guideline for the use of anti-D immunoglobulin for the prevention of haemolytic disease of the fetus and newborn. Transfus Med 2014;24:8-20.

16 Austin E, Bates S, de Silva M, Howarth D, Lubenko A, Rowley M, et al. Guidelines for the estimation of fetomaternal haemorrhage. Working Party of the British Committee for Standards in Haematology, Transfusion Taskforce, 2009. www.bcshguidelines.com/ documents/BCSH_FMH_bcsh_sept2009.pdf

\section{Accepted: 13 August 2014}

\section{Cite this as: BMJ 2014;349:95243}

This is an Open Access article distributed in accordance with the Creative Commons Attribution Non Commercial (CC BY-NC 3.0) license, which permits others to distribute, remix, adapt, build upon this work non-commercially, and license their derivative works on different terms, provided the original work is properly cited and the use is non-commercial. See: http://creativecommons.org/licenses/by-nc/3.0/. 


\section{Tables}

Table 1| Prediction of fetal RhD status by fetal RHD genotyping of cell-free DNA in maternal plasma compared with results of cord blood typing of RhD

\begin{tabular}{|c|c|c|c|c|c|c|}
\hline & \multicolumn{5}{|c|}{ Gestational age in completed weeks ${ }^{*}$} & \multirow[t]{2}{*}{ Total samples } \\
\hline & $<11$ & $11-13$ & $14-17$ & $18-23$ & $>24$ & \\
\hline Correct RhD negative & 337 & 341 & 225 & 321 & 696 & 1920 \\
\hline Correct RhD positive & 400 & 535 & 272 & 492 & 864 & 2563 \\
\hline False RhD negative & 16 & 1 & 1 & 1 & 0 & 19 \\
\hline False RhD positive & 1 & 4 & 1 & 5 & 7 & 18 \\
\hline $\begin{array}{l}\text { Inconclusive confirmed RhD } \\
\text { negative }\end{array}$ & 19 & 13 & 10 & 19 & 24 & 85 \\
\hline $\begin{array}{l}\text { Inconclusive confirmed RhD } \\
\text { positive }\end{array}$ & 92 & 62 & 33 & 50 & 71 & 308 \\
\hline Total & 865 & 956 & 542 & 888 & 1662 & 4913 \\
\hline $\begin{array}{l}\text { Sensitivity RhD positive (95\% } \\
\text { Cl)† }\end{array}$ & $\begin{array}{c}96.85 \text { (94.95 to } \\
98.05)\end{array}$ & $\begin{array}{l}99.83(99.06 \text { to } \\
99.97)\end{array}$ & $\begin{array}{l}99.67(98.17 \text { to } \\
99.94)\end{array}$ & 99.82 (9.96 to 99.97$)$ & $\begin{array}{c}100.00(99.59 \text { to } \\
100.00)\end{array}$ & $\begin{array}{l}99.34 \text { (98.98 to } \\
99.58)\end{array}$ \\
\hline $\begin{array}{l}\text { Specificity RhD positive }(95 \% \\
\text { Cl) }\end{array}$ & $\begin{array}{l}94.40 \text { (91.51 to } \\
96.34)\end{array}$ & $\begin{array}{l}95.25 \text { (92.53 to } \\
97.01)\end{array}$ & $\begin{array}{l}95.34 \text { (91.85 to } \\
97.38)\end{array}$ & $\begin{array}{l}93.04 \text { (89.86 to } \\
95.28)\end{array}$ & $\begin{array}{l}95.74 \text { (94.01 to } \\
96.98)\end{array}$ & $\begin{array}{l}94.91 \text { (93.86 to } \\
95.78)\end{array}$ \\
\hline
\end{tabular}

*Gestational ages shown are in completed postmenstrual weeks (for example, 11 weeks=11 weeks 0 days to 11 weeks +6 days).

†Assuming all inconclusive results are treated as RhD positive. 
Table 2| Impact of false negative results in terms of alloimmunisation, and risk to subsequent pregnancies

\begin{tabular}{|c|c|c|c|c|c|}
\hline & \multicolumn{5}{|c|}{ Gestational age in weeks } \\
\hline & $<11$ & $11-13$ & $14-17$ & $18-23$ & $>24$ \\
\hline False $\mathrm{RhD}$ negative $\%(\mathrm{Cl})$ & 1.85 (1.14 to 2.98$)$ & 0.10 (0.02 to 0.59$)$ & 0.18 (0.03 to 1.04$)$ & 0.11 (0.02 to 0.64$)$ & 0.00 (0 to 0.23$)$ \\
\hline $\begin{array}{l}\text { Risk of alloimmunisation per } 100000 \\
\text { pregnancies if cord blood typing is not } \\
\text { performed }(\mathrm{Cl})^{\star}\end{array}$ & 167 (103 to 268$)$ & 9.0 (2 to 53$)$ & 16.2 (3 to 94$)$ & 9.9 (2 to 58$)$ & $0(0$ to 20$)$ \\
\hline $\begin{array}{l}\text { Risk per } 100000 \text { pregnancies of having } \\
\text { subsequent severely affected child if cord } \\
\text { blood typing is not performed* } \dagger\end{array}$ & $7.4(4.6$ to 11.9$)$ & $0.4(0.08$ to 2.4$)$ & $0.72(0.1$ to 4.2$)$ & 0.440 .08 to 2.6 ) & $0(0$ to 0.9$)$ \\
\hline $\begin{array}{l}\text { Risk of alloimmunisation per } 100000 \\
\text { pregnancies if cord typing is performed in } \\
\text { pregnancies when cell-free fetal DNA result } \\
\text { is } R H D \text { negative }\end{array}$ & 19 (11 to 30$)$ & $1.0(0.2$ to 6$)$ & $1.8(0.3$ to 10$)$ & $1.1(0.2$ to 6$)$ & $0(0$ to 0.1$)$ \\
\hline $\begin{array}{l}\text { Risk per } 100000 \text { pregnancies of having } \\
\text { subsequent severely affected child if cord } \\
\text { blood typing is performed in pregnancies } \\
\text { when cell free fetal DNA result is } R H D \\
\text { negative* }\end{array}$ & $0.82(0.5$ to 1.3$)$ & $0.04(0.009$ to 0.26$)$ & 0.08 (0.01 to 0.46$)$ & $0.05(0.009$ to 0.28$)$ & $0(0$ to 0.11$)$ \\
\hline
\end{tabular}

*Assuming $9 \%$ alloimmunised if not given anti-RhD immunoglobulin at delivery (published range 4.3-9\%) and 1\% alloimmunised if given anti-RhD immunoglobulin at delivery based on cord RhD type.

†Assuming $62 \%$ have subsequent baby $71.5 \%$ of which are RhD positive, risk of death for affected baby is $5 \%$. Risk of permanent neurological damage in affected baby is $5 \%{ }^{8}$ 


\section{Figures}

Booking for antenatal care

Combined Down's syndrome screening test 2nd trimester Down's syndrome screening Anomaly scan

3rd trimester antibody check

$\square$ Recommended gestation $\square$ Extended gestation

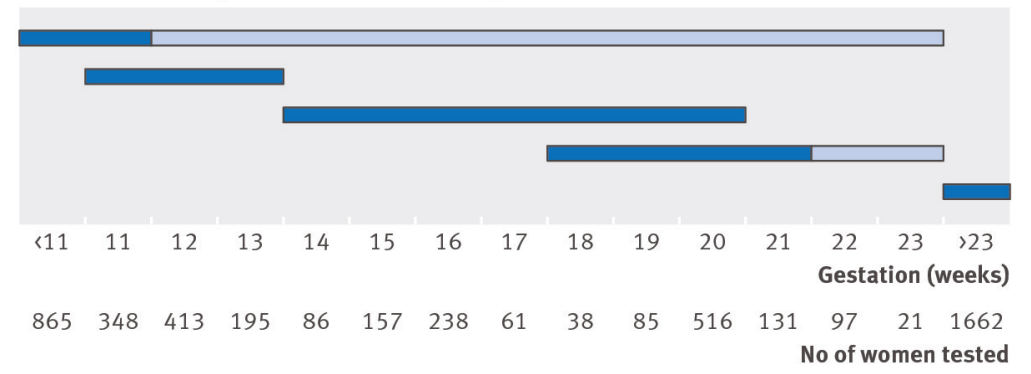

Fig 1 Maternity care pathway followed in England by gestational age together with number of women tested at each completed week of gestation. Dark bars indicate recommended gestations for tests but not all women book early in pregnancy and some attend late for their anomaly scan, so lighter bars show extended range of gestations, when women might have been recruited when attending for these indications

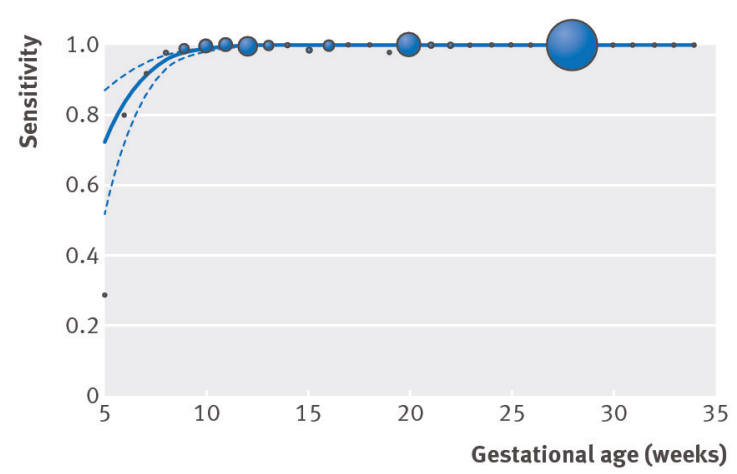

Fig 2 Sensitivity of determining $R H D$ positive fetuses according to gestational age (inconclusive results treated as $R H D$ positive). Fitted model (solid line) and 95\% bootstrap confidence intervals (dashed lines) obtained from generalised linear model incorporating random intercept to take into account repeat measures from same women over pregnancy. Weekly estimates are overlaid with point size proportional to number of women tested during each week 